\title{
Differentiating Tumor Progression from Pseudoprogression in Patients with Glioblastomas Using Diffusion Tensor Imaging and Dynamic Susceptibility Contrast MRI
}

S. Wang, M. Martinez-Lage, Y. Sakai, S. Chawla, S.G. Kim, M. Alonso-Basanta, R.A. Lustig, S. Brem, S. Mohan, R.L. Wolf, A. Desai, and

H. Poptani

\section{ABSTRACT}

BACKGROUND AND PURPOSE: Early assessment of treatment response is critical in patients with glioblastomas. A combination of DTI and DSC perfusion imaging parameters was evaluated to distinguish glioblastomas with true progression from mixed response and pseudoprogression.

MATERIALS AND METHODS: Forty-one patients with glioblastomas exhibiting enhancing lesions within 6 months after completion of chemoradiation therapy were retrospectively studied. All patients underwent surgery after MR imaging and were histologically classified as having true progression ( $>75 \%$ tumor), mixed response $(25 \%-75 \%$ tumor), or pseudoprogression $(<25 \%$ tumor). Mean diffusivity, fractional anisotropy, linear anisotropy coefficient, planar anisotropy coefficient, spheric anisotropy coefficient, and maximum relative cerebral blood volume values were measured from the enhancing tissue. A multivariate logistic regression analysis was used to determine the best model for classification of true progression from mixed response or pseudoprogression.

RESULTS: Significantly elevated maximum relative cerebral blood volume, fractional anisotropy, linear anisotropy coefficient, and planar anisotropy coefficient and decreased spheric anisotropy coefficient were observed in true progression compared with pseudoprogression $(P<$.05). There were also significant differences in maximum relative cerebral blood volume, fractional anisotropy, planar anisotropy coefficient, and spheric anisotropy coefficient measurements between mixed response and true progression groups. The best model to distinguish true progression from non-true progression (pseudoprogression and mixed) consisted of fractional anisotropy, linear anisotropy coefficient, and maximum relative cerebral blood volume, resulting in an area under the curve of 0.905 . This model also differentiated true progression from mixed response with an area under the curve of 0.901. A combination of fractional anisotropy and maximum relative cerebral blood volume differentiated pseudoprogression from nonpseudoprogression (true progression and mixed) with an area under the curve of 0.807 .

CONCLUSIONS: DTI and DSC perfusion imaging can improve accuracy in assessing treatment response and may aid in individualized treatment of patients with glioblastomas.

ABBREVIATIONS: $\mathrm{AUC}=$ area under the curve; $\mathrm{CL}=$ linear anisotropy coefficient; $\mathrm{CP}=$ planar anisotropy coefficient; $\mathrm{CS}=$ spheric anisotropy coefficient; $\mathrm{FA}=$ fractional anisotropy; $L R M=$ logistic regression model; $\max =$ maximum; $M D=$ mean diffusivity; PsP = pseudoprogression; $r C B V=$ relative cerebral blood volume; $\mathrm{TP}=$ true progression

$\mathrm{T}$ he current standard of care for newly diagnosed glioblastomas is surgical resection and concurrent temozolomide radiation therapy, followed by at least 6 months of adjuvant temozolomide. Treatment outcome is generally monitored by using standard clinical MR imaging based on accepted guidelines such as the

Received March 10, 2015; accepted after revision June 2.

From the Departments of Radiology (S.W., Y.S., S.M., R.L.W., H.P.), Division of Neuroradiology, Pathology and Laboratory Medicine (M.M.-L.), Radiation Oncology (M.A.-B., R.A.L.,), Neurosurgery (S.B.), and Hematology-Oncology (A.D.), Hospital of the University of Pennsylvania, Philadelphia, Pennsylvania; and Department of Radiology (S.C., S.G.K.), Center for Biomedical Imaging, New York University School of Medicine, New York, New York.

Current affiliation for H.P.: Department of Cellular and Molecular Physiology, University of Liverpool, UK.

This work was supported by National Institutes of Health grant 1R21CA170284. updated Response Assessment in Neuro-Oncology criteria. ${ }^{1,2}$ However, the appearance of enhancing lesions on MR imaging within the first 6 months after completion of chemoradiation therapy poses a challenge because it can reflect true progression (TP) or treatment-related changes known as pseudoprogression (PsP). PsP occurs in approximately a third of all patients with glioblastoma, ${ }^{3}$ in which lesions often decrease in size or stabilize without further treatment, resulting in a longer survival. Accurate identifica-

Please address correspondence to Harish Poptani, PhD, Department of Cellular and Molecular Physiology, University of Liverpool, 2/012 Nuffield Wing, Sherrington Building, Crown St, Liverpool L69 3BX, UK; e-mail: Harish.Poptani@liverpool.ac.uk

- Indicates open access to non-subscribers at www.ajnr.org

Indicates article with supplemental on-line photo.

http://dx.doi.org/10.3174/ajnr.A4474 
tion of PsP and TP is critical because patients with TP may require a change in therapeutic strategy while those with PsP may not. While published reports have attempted to differentiate PsP from TP, ${ }^{4-7}$ these studies did not account for the common finding of a mixture of treatment-related changes and recurrent tumor. Management of these partial responders may be challenging, with short-interval imaging studies often required to determine clinical course. If identified early, these patients may benefit from novel therapeutics.

Mean diffusivity (MD), measured from diffusion imaging, has been used to diagnose and monitor treatment response in brain tumors. ${ }^{8,9}$ Both mean and minimum MD values have been used in differentiating PsP from TP. ${ }^{4-6,10}$ However, due to the heterogeneity of treatment response, MD may have a limited role because reduced diffusion could represent not only highly cellular tumor areas but also inflammatory processes. ${ }^{9} \mathrm{DTI}$ is increasingly being used in the characterization of glioblastomas ${ }^{9,11}$; anisotropy measures, including fractional anisotropy (FA), linear anisotropy (CL), planar anisotropy (CP), and spheric anisotropy (CS), have been used to differentiate glioblastomas from metastasis ${ }^{1-13}$ and primary cerebral lymphomas. ${ }^{12,14}$ However, only 1 study has used DTI for differentiation of PsP and TP. ${ }^{15}$

Relative cerebral blood volume (rCBV) obtained from DSC perfusion imaging has been widely used for tumor grading, ${ }^{16}$ distinguishing recurrent tumor from radiation necrosis, ${ }^{17}$ and differentiating PsP from TP. ${ }^{18,19}$ Some studies have suggested that median rCBV and histogram analysis of rCBV can help differentiate PsP from TP. ${ }^{18,20-22}$ However, rCBV has not been used to identify a mixed or partial response.

The clinical management of patients with recurrent glioblastoma is rapidly changing because several alternative therapeutic options are being investigated, including bevacizumab, ${ }^{23}$ tumor treating fields, ${ }^{24}$ and immunotherapy. ${ }^{25}$ Increased incidence of PsP poses a dilemma for the treating physicians because determining the optimal therapeutic approach relies on a definitive diagnosis of TP, PsP, or mixed response. We hypothesize that DTI and DSC parameters have added value in making this differentiation and thus evaluated them for differentiating these 3 categories of treatment response.

\section{MATERIALS AND METHODS Patients}

This study was approved by the institutional review board and was compliant with the Health Insurance Portability and Accountability Act. MR imaging data from 41 patients with glioblastomas (14 women/27 men; $55.71 \pm 11.83$ years of age; age range, $23-80$ years), who had initially undergone gross total resection of the tumor followed by standard radiation therapy and temozolomide chemotherapy and exhibited new enhancing lesions on follow-up MR imaging within 6 months after completion of radiation therapy, were retrieved from the University of Pennsylvania data base from May 2011 to May 2014 and retrospectively analyzed. All patients underwent repeat surgery within 2 weeks after the MR imaging study in which the new enhancing lesions were first observed.

\section{MR Imaging Data Acquisition}

MR imaging studies were performed on a Tim Trio 3T wholebody scanner (Siemens, Erlangen, Germany) by using a 12-channel phased array head coil. Routine sequences included axial T1- weighted 3D MPRAGE $(\mathrm{TR} / \mathrm{TE} / \mathrm{TI}=1760 / 3.1 / 950 \mathrm{~ms}, 192 \times 256$ matrix size, 1-mm section thickness) and axial FLAIR (TR/TE/ $\mathrm{TI}=9420 / 141 / 2500 \mathrm{~ms}, 3-\mathrm{mm}$ section thickness). DTI data were acquired by using a single-shot spin-echo EPI sequence with parallel imaging by using generalized autocalibrating partially parallel acquisition and an acceleration factor of 2. Diffusion weighting was applied in 30 isotropically distributed directions by using a b-value of $1000 \mathrm{~s} / \mathrm{mm}^{2}$, with a total acquisition time of 8 minutes. For DSC imaging, a bolus of gadobenate dimeglumine (MultiHance; Bracco Diagnostics, Princeton, New Jersey) was injected with a preloading dose of $0.07 \mathrm{mmol} / \mathrm{kg}$, which was used to reduce the effect of contrast agent leakage on CBV measurements. ${ }^{26}$ DSC imaging was performed by using a gradient-echo echo-planar imaging sequence during a second $0.07-\mathrm{mmol} / \mathrm{kg}$ bolus of contrast agent $\left(\mathrm{TR} / \mathrm{TE}=2000 / 45 \mathrm{~ms}, \mathrm{FOV}=22 \times 22 \mathrm{~cm}^{2}\right.$, resolution $=$ $1.72 \times 1.72 \times 3 \mathrm{~mm}^{3}, 20$ sections, 45 measurements with at least 10 image volumes before bolus arrival, acquisition time $=1 \mathrm{~min}$ ute 38 seconds). The injection rate was $5 \mathrm{~mL} / \mathrm{s}$ for all patients and was immediately followed by a bolus injection of saline (total of 20 $\mathrm{mL}$ at the same rate). Postcontrast T1-weighted 3D MPRAGE images were acquired after completion of the DSC sequence.

\section{Image Processing}

The diffusion tensor datasets were coregistered to the $b=0 \mathrm{~s} / \mathrm{mm}^{2}$ images by using a 3D affine transformation estimated by maximizing the mutual information between the images. ${ }^{13}$ The corrected raw images were combined to estimate the DTI parametric maps by using in-house software (IDL; ITT Visual Information Solutions, Boulder, Colorado). Pixel-wise MD, FA, CL, CP, and CS maps were computed by using the methods described earlier. ${ }^{11,12}$ Leakage-corrected CBV maps using the $\gamma$ variate function were generated by using NordicICE software (NordicNeuroLab, Bergen, Norway).

The DTI, CBV maps, and FLAIR images were coregistered to contrast-enhanced T1-weighted images. The CBV maps were normalized to the contralateral normal white matter to generate rCBV.A semiautomatic segmentation approach was used to generate a mask from the enhancing region by using the methods described earlier. ${ }^{11-13}$ The median DTI metrics and rCBV values from the enhancing region were measured. In addition, the lower 10th percentile MD values were measured from the enhancing region and reported as minimum MD. ${ }^{27}$ The top 90th percentile rCBV values were measured from the enhancing region and reported as $\mathrm{rCBV}_{\max }{ }^{12}$ Data analysis tools, including DTI computing, image coregistration, and segmentation, were implemented by using IDL routines. The total time for postprocessing was approximately 2 hours.

\section{Histologic Analysis}

Pathologic samples were originally cut, mounted, and stained with hematoxylin-eosin by standard methods. Immunohistochemistry for Ki-67 (mouse monoclonal, MIB-1, IR62661; Dako, Carpinteria, California) and p53 (mouse monoclonal, 1:60; DO-7, M7001; Dako) was performed by using a Bond III automated system (Leica Biosystems, Buffalo Grove, Illinois). The entirety of submitted material for each case was examined by a board-certified neuropathologist (M.M.-L.) who was blinded to the results of the MR imaging studies. The slides were examined to determine the relative degree of recurrent glioma and treatment-related changes. The percentage of geo- 

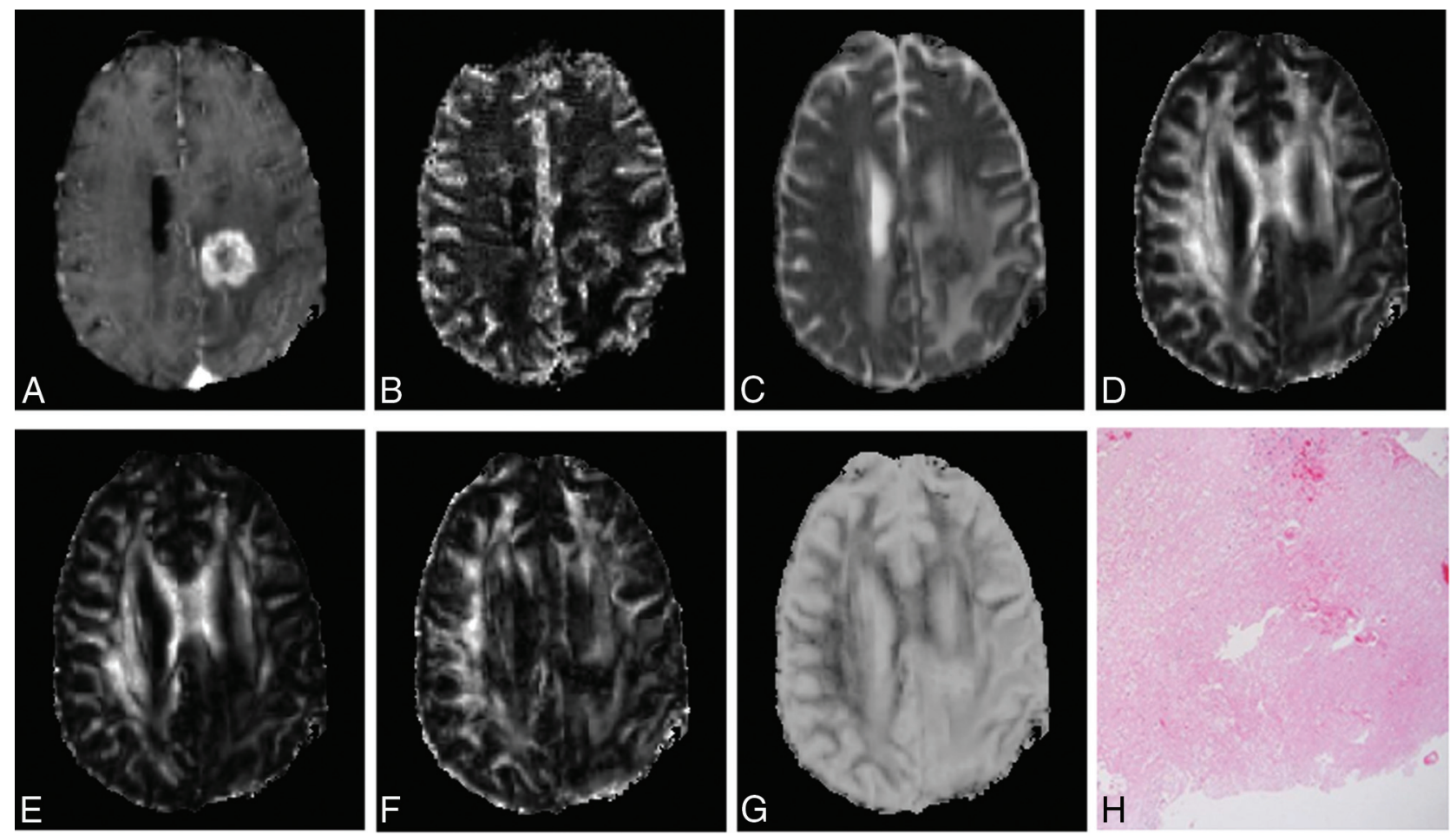

FIG 1. Axial MR images of a 44-year-old man with PsP. Contrast-enhanced TT-weighted image $(A)$ shows a new enhancing lesion in the left parietal lobe. CBV map $(B)$ shows moderately increased CBV from the lesion. $M D(C)$ looks similar to the normal white matter. Decreased $F A(D), C L(E)$, and $C P(F)$ and increased CS $(G)$ are observed from the enhancing part compared with normal white matter. Photomicrograph of a histologic section $(H$, hematoxylineosin stain, 50X magnification) reveals most of the tissue with treatment-related changes, including extensive geographic necrosis and vascular fibrinoid necrosis $(90 \%)$.

graphic tissue necrosis across the specimen was assessed in an initial approach, excluding normal or quasinormal brain parenchyma. Histologic features associated with treatment, including vascular necrosis, hyalinization, hemosiderin, lymphocyte and macrophage infiltrates, gliosis, fibrosis, and dystrophic calcification, were also documented. Tumor-specific characteristics within the specimen, including neoplastic high cellularity, the presence of pseudopalisading necrosis, endothelial cell proliferation, and increased mitotic activity were additionally used to either increase or decrease the weightage for the presence of overall malignant features. The patients were grouped in 3 categories: $<25 \%$ malignant features, PsP ( 8 patients; 3 women/5 men; $48.5 \pm 12.72$ years of age); $25 \%-75 \%$ malignant features, mixed tumor with treatment response ( 12 patients; 5 women $/ 7$ men; $58.25 \pm 7.47$ years of age); and $>75 \%$ malignant features, TP (21 patients; 6 women/15 men; $57.0 \pm 12.9$ years of age). Strong and diffuse nuclear staining for $\mathrm{p} 53$ was used as supportive evidence of the presence of tumor, but the lack of staining did not reject the presence of recurrent glioma. We calculated the proliferative index with Ki-67 for each case as a percentage of positive tumor cells, avoiding areas of inflammatory infiltrates.

\section{Statistical Analysis}

A Mann-Whitney $U$ test was used to compare the difference in the median $\mathrm{MD}, \mathrm{FA}, \mathrm{CL}, \mathrm{CP}, \mathrm{CS}$, and $\mathrm{rCBV}$ values and minimum $\mathrm{MD}$ and $\mathrm{rCBV}_{\max }$ among PsP, mixed, and TP groups. Bonferroni correction was used to adjust for multiple comparisons, and a $P$ value $<.05$ was considered significant. A multivariate logistic regression model (LRM) was used to determine the best classification model, and a leave-one-out cross-validation approach was applied to estimate the accuracy of the LRM. Areas under the receiver operating characteristic curves (AUCs) were computed by using the selected parameters and the LRM output. A cutoff value for each parameter was determined by maximizing the sum of sensitivity and specificity. All statistical analyses were conducted by using PASW Statistics, Version 18 (IBM, Armonk, New York).

\section{RESULTS}

Representative MR images and histologic photomicrographs from patients with PsP, mixed, and TP features are shown in Figs 1-3, respectively. MD maps demonstrate variable degrees of diffusivity from the enhancing parts of the tumor. Anisotropy and rCBV maps also show variability. However, quantitative analysis revealed significantly higher median FA, CL, and CP values and higher rCBV in patients with TP compared with PsP and mixed response (Fig 4).

A pair-wise comparison of DTI and DSC parameters from enhancing tissue is shown in Fig 4. While median MD values did not show significant differences between groups $(P>.05)$, significantly higher $\mathrm{rCBV}_{\max }(4.75$ versus $2.90, P=.007)$, FA (0.14 versus $0.11, P=.008), \mathrm{CL}(0.05$ versus $0.04, P=.04)$, and $\mathrm{CP}$ (0.08 versus $0.06, P=.002)$, and decreased CS ( 0.87 versus 0.90 , $P=.004)$ were observed in TP compared with PsP. There were also significant differences between mixed and TP groups in rCB$\mathrm{V}_{\max }(4.75$ versus $3.31, P=.02)$, $\mathrm{FA}(0.14$ versus $0.11, P=.01)$, $\mathrm{CP}(0.08$ versus $0.06, P=.001)$, and CS $(0.87$ versus $0.89, P=.02)$ measurements. None of the parameters demonstrated a significant difference between PsP and mixed response. Of all the parameters, $\mathrm{CP}$ and CS showed a significant difference between PsP 

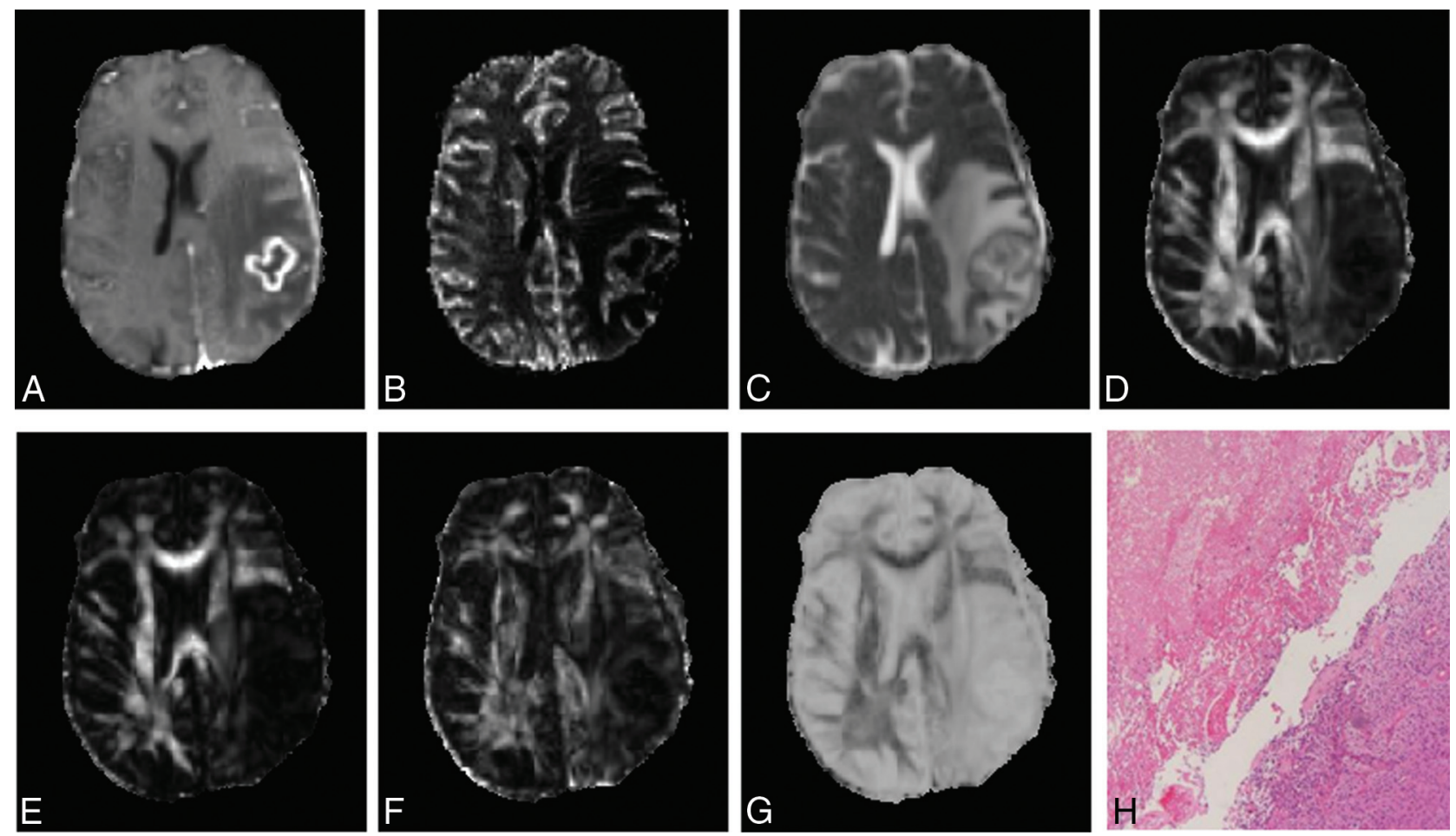

FIG 2. Axial images from the brain of a 59-year-old woman with mixed features of response, including areas of treatment-related changes and TP. Contrast-enhanced T1-weighted image $(A)$ shows a new enhancing lesion in the left parietal lobe. The lesion shows slightly elevated perfusion on the CBV map $(B)$ and lower MD $(C)$. Lower FA $(D), C L(E), C P(F)$, and higher $C S(G)$ values from the enhancing part are noticed relative to the normal white matter. The imaging appearance looks similar to that of PsP (Fig 1). Photomicrograph of a histologic section ( $H$, hematoxylin-eosin stain, $50 \times$ magnification) has similar amounts of treatment-related changes (50\%) and viable tumor (50\%).
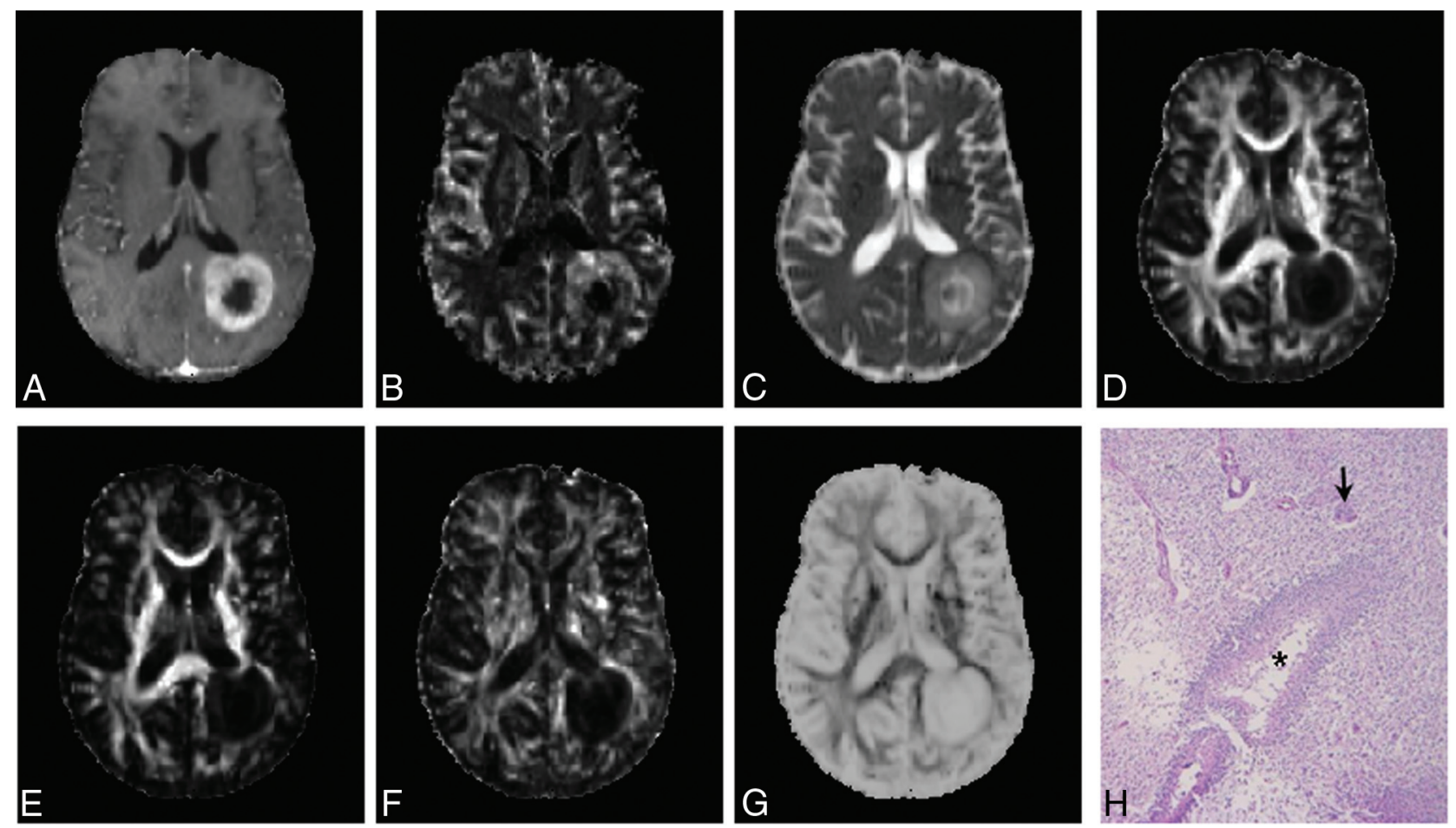

FIG 3. Axial brain images from a 54-year-old man showing TP. Contrast-enhanced T1-weighted image $(A)$ shows a ring-enhancing lesion in the left parietal lobe. High rCBV $(B)$ and increased $M D(C)$ are observed from the lesion. The enhancing part of the lesion demonstrates decreased $F A(D), C L(E)$, and $C P(F)$ and increased CS $(G)$. Findings in a photomicrograph of a histologic section $(H$, hematoxylin-eosin stain, $50 \times$ magnification) are similar to the patient's de novo glioblastoma, with areas of high tumor cellularity, pseudopalisading necrosis (asterisks), and endothelial proliferation (arrow) and increased mitotic activity. 

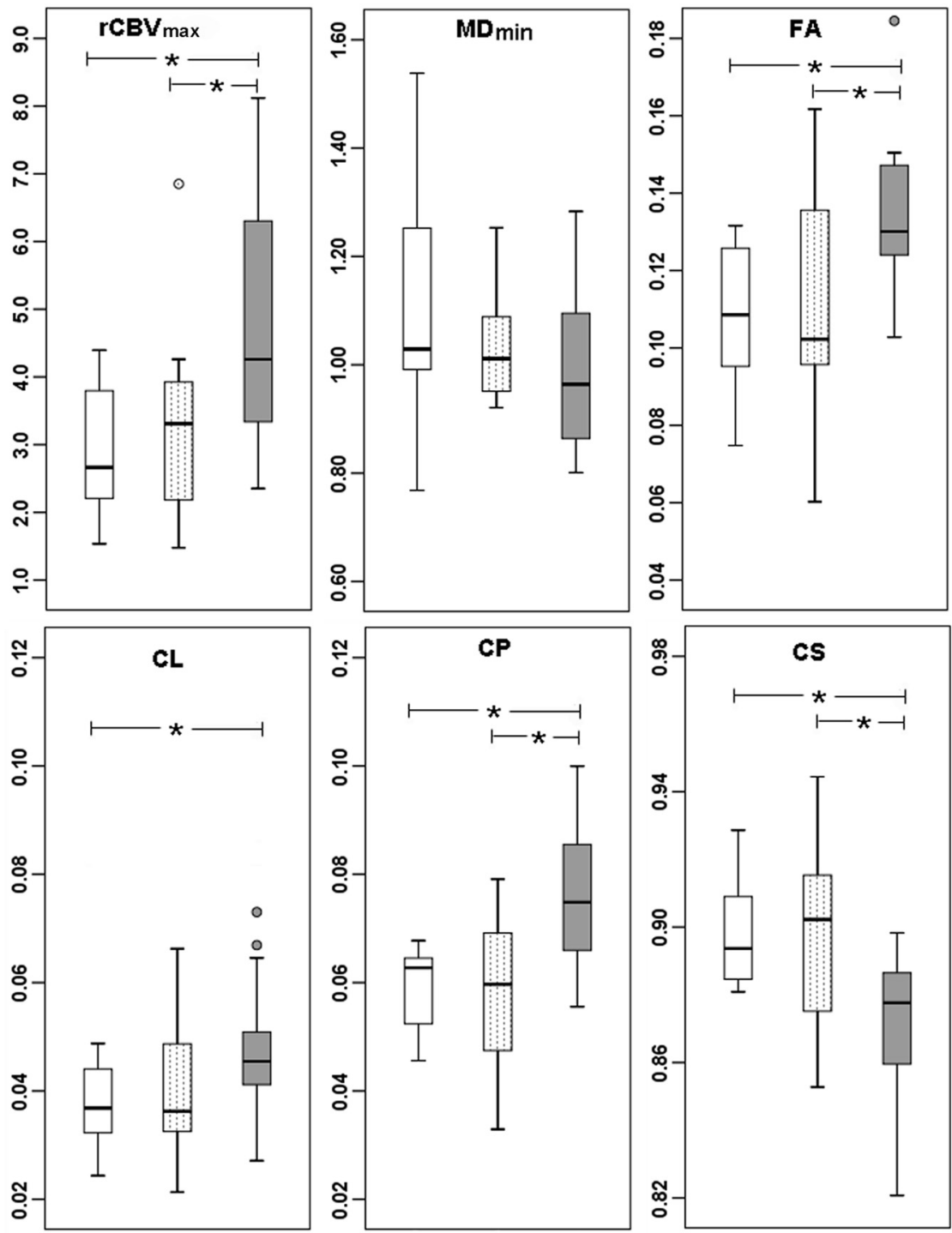

FIG 4. Boxplots of diffusion (minimum MD, FA, CL, CP, and CS) and perfusion (maximum rCBV) characteristics for patients with posttreatment glioblastomas in TP (gray), PsP (white), and mixed tumor (dotted). The solid line inside each box represents the median value, while the edges represent the 25th and 75th percentiles. The straight line (bars) on each box indicates the range of data distribution. Circles represent outliers (values $>1.5$ box length from the 75 th/25th percentiles). The asterisk indicates a significant difference $(P<.05)$ for group comparison.

and TP, and CP showed a significant difference between mixed and TP after Bonferroni correction $(P<.006)$.

\section{Discrimination of TP versus PsP and Mixed Response}

The discrimination analysis was first performed to distinguish TP from $\mathrm{PsP}$ and mixed response. CP was the single best predictor for classification (AUC $=0.84$ ), followed by $\mathrm{FA}$ and $\mathrm{rCBV}_{\max }$ (AUC $=0.78$, Table). The imaging parameters were then used for a multivariate logistic regression analysis with backward stepwise selection, which indicated that the best classification of TP from non-TPs, including PsP and mixed, was achieved with 3 parameters, $\mathrm{FA}, \mathrm{CL}$, and $\mathrm{rCBV}_{\max }$, as follows:

$$
\begin{aligned}
& f\left(F A, C L, r C B V_{\max }\right) \\
& \quad=\frac{1}{1+\exp \left[-\left(\beta_{0}+\beta_{1} F A+\beta_{2} C L+\beta_{3} r C B V_{\max }\right)\right]},
\end{aligned}
$$

where $\beta_{0}=-16.17, \beta_{1}=194.01, \beta_{2}=$ -285.65 , and $\beta_{3}=1.21$. Figure $5 A$ shows the receiver operating characteristic curves for the best LRM and selected parameters. The cutoff value for the LRM was 0.55 with a sensitivity $=76 \%$, specificity $=95 \%$, and AUC $=0.905$ (Table). Leave-one-out cross-validation analysis revealed that $78 \%$ of cases were correctly classified by using the LRM. Although CP was the single best predictor, it was not selected by the statistical model for highest sensitivity. Using forward stepwise selection, the best model included CP and $\mathrm{rCBV}_{\text {max }}$, resulting in an AUC of 0.89, similar to the model FA, CL, and $\mathrm{rCBV}_{\text {max. }}$. In addition, there was a high correlation $(r=0.84)$ between FA and CP (On-line Figure), suggesting that the results from either model were similar.

\section{Discrimination of PsP versus TP and Mixed Response}

The single best predictor for classification was $\mathrm{CP}$ (AUC $=0.74$ ), followed by $\mathrm{rCBV}_{\text {max }}(\mathrm{AUC}=0.73)$ and $\mathrm{FA}(\mathrm{AUC}=$ 0.70 , Table). The best LRM for classification of PsP from non-PsPs, including TP and mixed response, was achieved with 2 parameters, $\mathrm{FA}$ and $\mathrm{rCBV}_{\max }$, as follows:

$$
\begin{aligned}
& f\left(F A, r C B V_{\max }\right) \\
& \begin{array}{c}
=\frac{1}{1+\exp \left[-\left(\beta_{0}+\beta_{1} F A\right.\right.}, \\
\left.\left.+\beta_{2} r C B V_{\max }\right)\right]
\end{array}
\end{aligned}
$$

where $\beta_{0}=-3.59, \beta_{1}=23.52$, and $\beta_{2}=0.62$. Figure $5 B$ shows the receiver operating characteristic curves for the best LRM and selected parameters. The cutoff value for the LRM was 0.77 with sensitivity $=79 \%$, specificity $=75 \%$, and AUC $=$ 0.807 (Table). Leave-one-out cross-validation analysis revealed that $63.4 \%$ of cases were correctly classified by using the LRM.

\section{Discrimination between TP and Mixed Response}

A subanalysis was performed to differentiate TP from mixed response so that the patients with mixed response could be closely monitored with short-interval imaging scans or enrolled in novel therapeutic trials. $\mathrm{CP}$ was again the single best predictor for classification $(\mathrm{AUC}=0.83)$, followed by FA $(\mathrm{AUC}=0.76)$ and $\mathrm{rCBV}_{\max }$ $($ AUC $=0.74$, Table). The LRM for classification of TP from mixed response was achieved with 3 parameters, including FA, CL, and $\mathrm{rCBV}_{\text {max }}$, as follows:

$$
f\left(F A, C L, r C B V_{\max }\right)
$$

$$
=\frac{1}{1+\exp \left[-\left(\beta_{0}+\beta_{1} F A+\beta_{2} C L+\beta_{3} r C B V_{\max }\right)\right]},
$$


Sensitivity, specificity, and cutoff values of best models for classification $^{a}$

\begin{tabular}{|c|c|c|c|c|c|}
\hline Model & Sensitivity & Specificity & $\begin{array}{l}\text { Cutoff } \\
\text { Values }\end{array}$ & AUC & $95 \% \mathrm{Cl}$ \\
\hline \multicolumn{6}{|l|}{ TP vs PsP + mixed } \\
\hline$C P$ & 0.71 & 0.90 & 0.07 & 0.84 & $0.72-0.96$ \\
\hline FA & 0.71 & 0.75 & 0.13 & 0.78 & $0.64-0.93$ \\
\hline $\mathrm{CL}$ & 0.71 & 0.75 & 0.04 & 0.72 & $0.56-0.88$ \\
\hline $\mathrm{rCBV}_{\max }$ & 0.62 & 0.80 & 4.06 & 0.77 & $0.63-0.92$ \\
\hline $\mathrm{FA}+\mathrm{CL}+\mathrm{rCBV}_{\max }$ & 0.76 & 0.95 & 0.55 & 0.90 & $0.81-1.00$ \\
\hline \multicolumn{6}{|l|}{ PsP vs TP + mixed } \\
\hline$C P$ & 0.57 & 1.00 & 0.07 & 0.74 & $0.59-0.89$ \\
\hline FA & 0.40 & 1.00 & 0.13 & 0.70 & $0.52-0.87$ \\
\hline $\mathrm{rCBV}_{\text {max }}$ & 0.82 & 0.63 & 2.77 & 0.73 & $0.54-0.91$ \\
\hline $\mathrm{FA}+\mathrm{rCBV}_{\max }$ & 0.79 & 0.75 & 0.77 & 0.81 & $0.66-0.95$ \\
\hline \multicolumn{6}{|l|}{ TP vs mixed } \\
\hline $\mathrm{CP}$ & 0.71 & 0.83 & 0.07 & 0.83 & $0.69-0.97$ \\
\hline FA & 0.71 & 0.75 & 0.13 & 0.76 & $0.56-0.95$ \\
\hline $\mathrm{CL}$ & 0.71 & 0.75 & 0.04 & 0.69 & $0.49-0.90$ \\
\hline $\mathrm{rCBV}_{\text {max }}$ & 0.62 & 0.83 & 4.06 & 0.74 & $0.57-0.92$ \\
\hline $\mathrm{FA}+\mathrm{CL}+\mathrm{rCBV}_{\max }$ & 0.76 & 1.00 & 0.65 & 0.90 & $0.78-1.00$ \\
\hline \multicolumn{6}{|l|}{ PsP vs mixed } \\
\hline$M D_{\min }$ & 0.50 & 0.88 & 0.98 & 0.62 & $0.34-0.89$ \\
\hline
\end{tabular}

where $\beta_{0}=-15.43, \beta_{1}=202.14, \beta_{2}=-313.99$, and $\beta_{3}=1.20$. Figure $5 C$ shows the receiver operating characteristic curves for the best LRM and selected parameters. The cutoff value for the LRM was 0.65 with sensitivity $=76 \%$, specificity $=100 \%$, and AUC $=0.901$ (Table). Leave-one-out cross-validation analysis revealed that $72.7 \%$ of cases were correctly classified by using the LRM.

\section{Discrimination of PsP from Mixed Response}

A final analysis to differentiate patients with PsP from those with mixed response showed a significant overlap between the 2 groups, with only $\mathrm{MD}$ having some predictive value with an $\mathrm{AUC}=0.62$ followed by $\mathrm{rCBV}_{\max }(\mathrm{AUC}=0.57)$.

\section{DISCUSSION}

The heterogeneity and variability in response did not allow differentiating TP from PsP simply by visual inspection of the parametric maps. However, a quantitative analysis of DTI parameters and $\mathrm{rCBV}_{\max }$ from the enhancing regions of the lesion demonstrated better assessment of treatment response in patients with glioblastomas. Such a categorization is clinically feasible because the postprocessing time was only approximately 2 hours, indicating that our proposed analytic approach may aid in individualized treatment management and better clinical decision-making.

\section{Identification of TP}

Early identification of TP could prevent further delays in repeat surgery or enrollment in alternative clinical trials. The LRM analysis indicated that the best model to distinguish TP from PsP or mixed responses was based on FA, CL, and $\mathrm{rCBV}_{\text {max }}$. Higher anisotropy values have been reported in glioblastomas compared with brain metastases and primary cerebral lymphomas. ${ }^{1-13}$ High FA in glioblastomas is probably related to the orientation of overproduced extracellular matrix. ${ }^{1,28}$ Glioblastoma tumor cells produce large amounts of tumor-specific extracellular matrix components, which can serve as a substrate for adhesion and subsequent migration of the tumor cells through the enlarged extra- cellular space, ${ }^{28}$ which may explain the elevated anisotropy observed in patients with TP. However, a previous study reported no difference in anisotropy measures between PsP and TP. ${ }^{15}$ Potential reasons for this discrepancy may be because we divided the patients on the basis of the histologic features as opposed to grouping on the basis of follow-up imaging used in the previous study. ${ }^{15}$ In addition, we assessed 3 categories, including mixed response, instead of just separating PsP and TP.

DSC imaging can be helpful in differentiating tumor recurrence from radiation necrosis. ${ }^{17,29}$ Recent studies have also used DSC imaging ${ }^{18,19,21}$ to detect TP from PsP. Kong et al ${ }^{18}$ reported that a mean rCBV value of 1.47 had $81.5 \%$ sensitivity and $77.8 \%$ specificity in differentiating PsP from TP, while Kim et $\mathrm{al}^{20}$ reported a histogram analysis of $\mathrm{rCBV}$, in which a peak height position of 1.7 showed $90.2 \%$ sensitivity and $91.1 \%$ specificity for differentiating tumor recurrence from treatment changes. In comparison, we used $\mathrm{rCBV}_{\max }$ and observed that a threshold $\mathrm{rCBV}_{\max }$ value of 4.06 led to a sensitivity of $62 \%$ and specificity of $80 \%$ in differentiating TP from PsP and mixed tumors.

\section{Identification of PsP}

Accurate identification of PsP is critical for patient management because unnecessary repeat surgery/biopsy can be avoided in these patients and they can continue on an effective temozolomide regimen with standard imaging follow-up of 3-6 months, thereby reducing patient care costs. Logistic regression analysis showed that the best model to differentiate PsP from TP and mixed response included FA and $\mathrm{rCBV}_{\text {max }}$.

Pseudoprogression is predominantly a subacute treatment-related reaction. Pathologically, it corresponds to gliosis and radiation-induced reactive changes including disruption of the BBB, inflammation, increased permeability, and edema. These changes cause increased enhancement on MR imaging and can mimic TP. ${ }^{9}$ Several studies have reported that PsP exhibits higher MD values from the enhancing region than $\mathrm{TP}$, partly due to the extent of cellular death and vascular changes in PsP. ${ }^{4,6}$ Minimum MD values have been reported to be prognostic of outcomes in gliomas. ${ }^{30} \mathrm{Chu}$ et $\mathrm{al}^{5}$ reported that the fifth percentile of the cumulative MD histogram was the most promising parameter in the differentiation of TP and PsP. Although we found a similar trend for minimum MD, calculated from the 10th percentile of the MD value, it did not reach statistical significance. There are a limited number of studies regarding the role of FA in the evaluation of treatment response. ${ }^{15,31} \mathrm{Xu}$ et $\mathrm{al}^{31}$ reported a low FA ratio in radiation necrosis compared with recurrent tumor. rCBV measurements have also been used in identifying PsP. ${ }^{9,32}$ The inflammation/necrotic processes involved in PsP result in lower rCBV values, ${ }^{9,32}$ and our results confirm this hypothesis. In addition, our study showed that a combination of FA and $\mathrm{rCBV}_{\max }$ can help in identifying PsP from TP or mixed response.

\section{Identification of Mixed Response}

On a practical level, posttreatment new enhancing lesions usually contain a mixture of viable neoplasm and treatment-induced changes, and a more accurate assessment of the relative contribution of each entity can guide clinical decision-making. However, 
TP vs PsP+Mixed
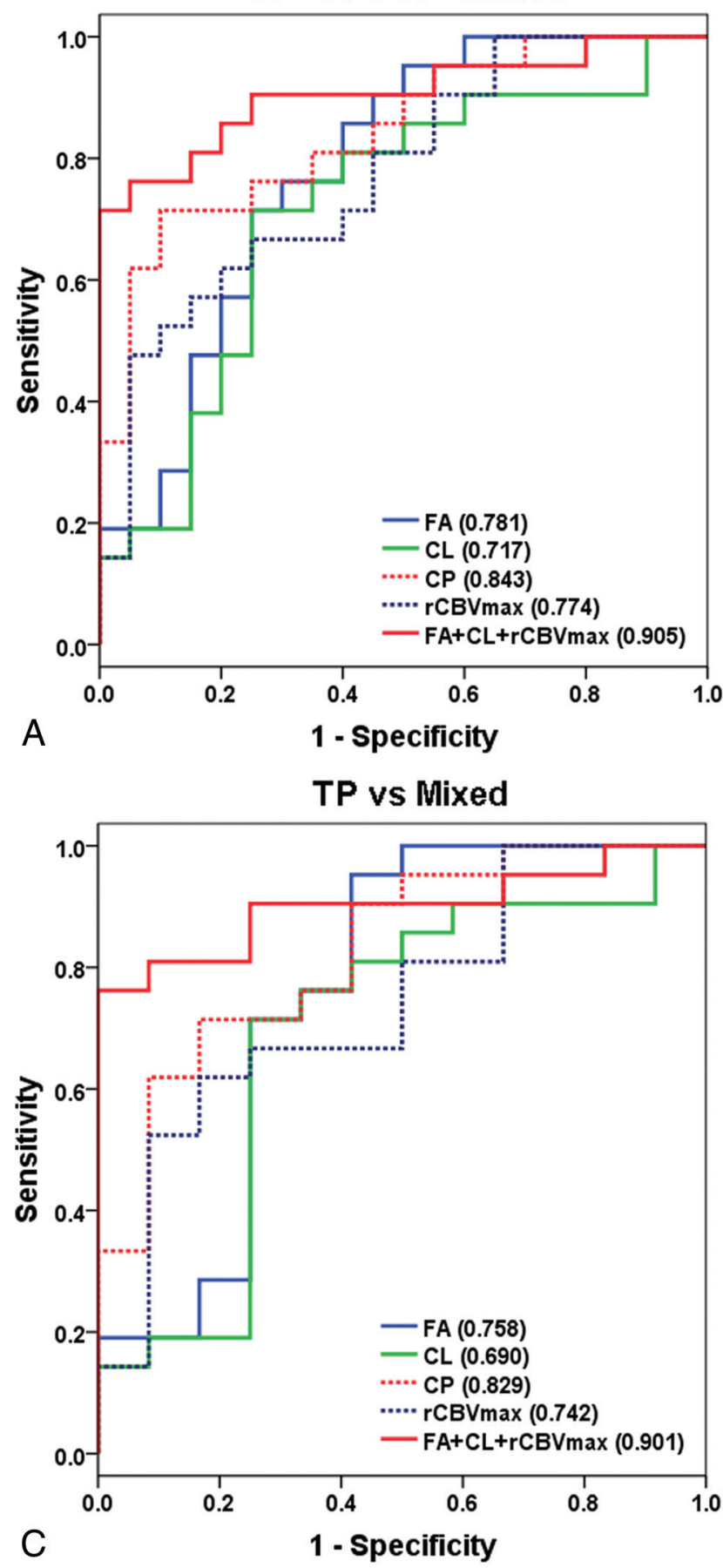

PsP vs TP+Mixed
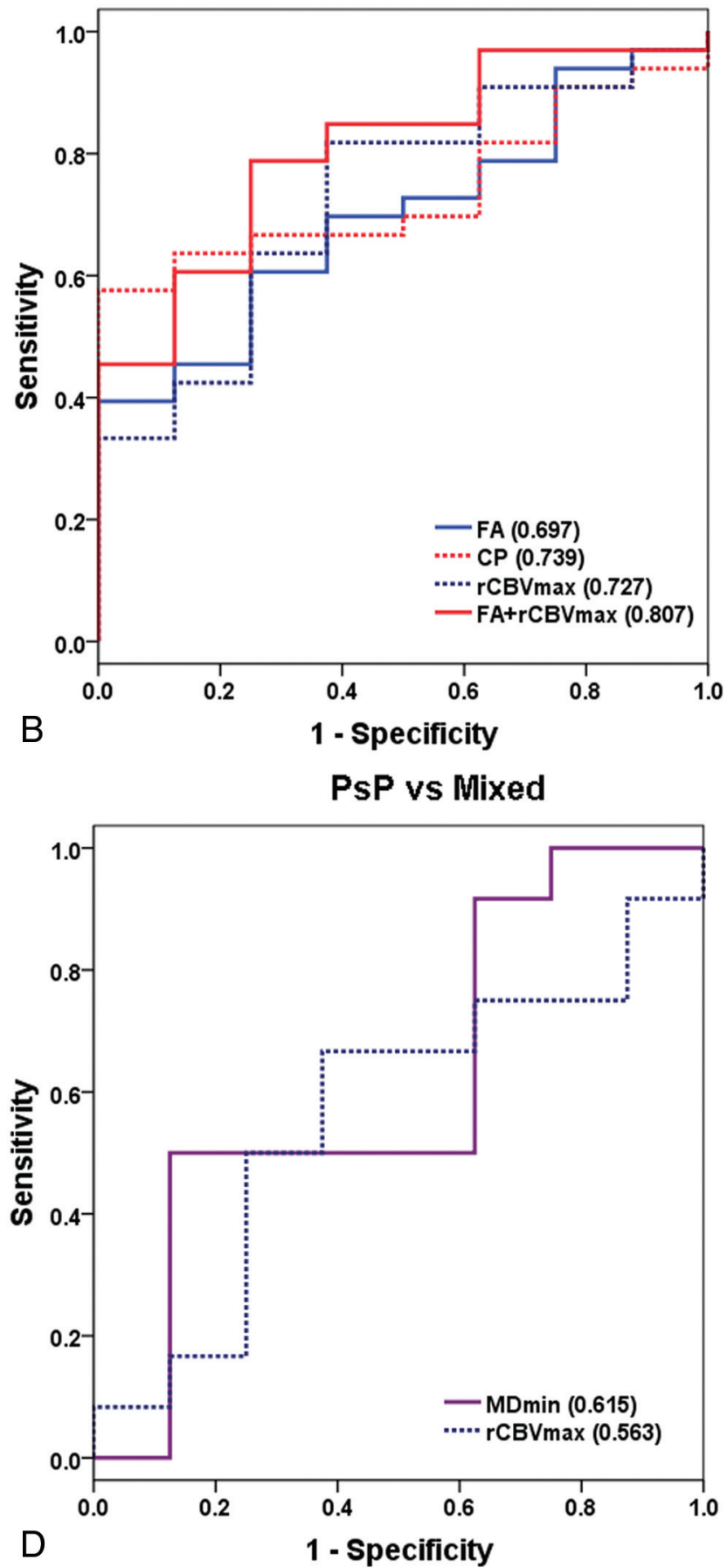

FIG 5. Receiver operating characteristic curves of the imaging parameters for identifying different groups. The numbers in the parentheses show AUC values. The logistic regression model of $\mathrm{FA}, \mathrm{CL}$, and $\mathrm{rCBV}_{\max }$ from the enhancing part of the tumor was the best predictor for differentiation of TP from non-TP, including PsP and mixed response with an AUC $=0.905(A)$. This model can also

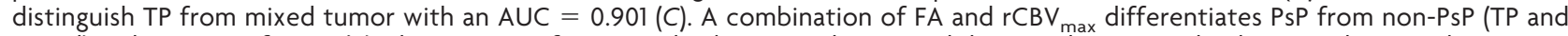
mixed) with an AUC of $0.807(B)$. There is a significant overlap between the PsP and the mixed group, and only MD is shown to have some predictive value with an $A \cup C=0.615(D)$.

most previous studies have attempted to only differentiate between PsP and TP. ${ }^{4,6,15,19}$ DTI and DSC imaging findings between viable neoplasm and treatment-induced changes are variable and difficult to synthesize on routine visual inspection. We believe the quantitative analysis of imaging parameters ${ }^{8,33}$ should yield a better estimate of each component. Our findings indicate that DTI and perfusion may have a complementary predictive value for the evaluation of treatment response and demonstrate that a combination of $\mathrm{FA}, \mathrm{CL}$ and $\mathrm{rCBV}_{\max }$ can differentiate mixed response from TP with high sensitivity. Although similar trends were observed between PsP and mixed response, DTI and DSC parameters had relatively modest utility in distin- 
guishing mixed response from PsP. Because dynamic contrastenhanced MR imaging is increasingly being used to assess brain tumors, we believe future studies including quantitative dynamic contrast-enhanced-based parameters may further enhance the sensitivity of our method in differentiating PsP from mixed response.

While these results are promising, the study has some limitations, including a retrospective analysis design and a relatively smaller sample size. In addition, image-guided biopsy may be necessary to evaluate the pathophysiologic basis of higher CP and FA in TP. A clinical follow-up study by using progression-free or overall analysis as end points for determination of PsP and TP at the time of initial MR imaging is desirable because not all patients will undergo repeat surgery for confirmation of imaging findings.

\section{CONCLUSIONS}

Our study shows that a combination of DTI and DSC perfusion parameters can help in the evaluation of treatment response in glioblastomas and may aid in the optimal management of these patients.

\section{ACKNOWLEDGMENTS}

We acknowledge Tianyu Yin for assistance in data analysis; Dr Ruyun Jin, Medical Data Research Center, Providence Health \& Services, Portland, Oregon, for statistical analysis; and research coordinators Lisa Desiderio, Katelyn Reilly, and Krista Huff.

Disclosures: Maria Martinez-Lage_RELATED: National Institutes of Health (Principal Investigator: Harish Poptani). Suyash Mohan—RELATED: Grant: National Institutes of Health (R21 grant).* Ronald L. Wolf-RELATED: Grant: National Institutes of Health,* Comments: 5-R21-CA-170284-02 direct/indirect support. I get a small amount of salary support under this grant $(<\$ 10,000)$; previous Principal Investigator $\mathrm{H}$. Poptani, but now I am the Principal Investigator on behalf of the University of Pennsylvania. Arati Desai-RELATED: Grant: National Institutes of Health (R21). ${ }^{*}$ Harish Poptani-RELATED: Grant: National Institutes of Health*; UNRELATED: Consultancy: American College of Radiology Image Metrix, Comments: consulting for MRS study trials; Grants/Grants Pending: National Institutes of Health. * * Money paid to the institution.

\section{REFERENCES}

1. Macdonald DR, Cascino TL, Schold SC Jr, et al. Response criteria for phase II studies of supratentorial malignant glioma. J Clin Oncol 1990;8:1277-80 Medline

2. Wen PY, Macdonald DR, Reardon DA, et al. Updated response assessment criteria for high-grade gliomas: response assessment in neuro-oncology working group. J Clin Oncol 2010;28:1963-72 CrossRef Medline

3. Topkan E, Topuk S, Oymak E, et al. Pseudoprogression in patients with glioblastoma multiforme after concurrent radiotherapy and temozolomide. Am J Clin Oncol 2012;35:284-89 CrossRef Medline

4. Cha J, Kim ST, Kim HJ, et al. Differentiation of tumor progression from pseudoprogression in patients with posttreatment glioblastoma using multiparametric histogram analysis. AJNR Am J Neuroradiol 2014;35:1309-17 CrossRef Medline

5. Chu HH, Choi SH, Ryoo I, et al. Differentiation of true progression from pseudoprogression in glioblastoma treated with radiation therapy and concomitant temozolomide: comparison study of standard and high-b-value diffusion-weighted imaging. Radiology 2013;269:831-40 CrossRef Medline

6. Lee WJ, Choi SH, Park CK, et al. Diffusion-weighted MR imaging for the differentiation of true progression from pseudoprogression following concomitant radiotherapy with temozolomide in pa- tients with newly diagnosed high-grade gliomas. Acad Radiol 2012; 19:1353-61 CrossRef Medline

7. Suh CH, Kim HS, Choi YJ, et al. Prediction of pseudoprogression in patients with glioblastomas using the initial and final area under the curves ratio derived from dynamic contrast-enhanced T1weighted perfusion MR imaging. AJNR Am J Neuroradiol 2013;34: 2278-86 CrossRef Medline

8. Hamstra DA, Chenevert TL, Moffat BA, et al. Evaluation of the functional diffusion map as an early biomarker of time-to-progression and overall survival in high-grade glioma. Proc Natl Acad Sci U S A 2005;102:16759-64 CrossRef Medline

9. Hygino da Cruz LC Jr, Rodriguez I, Domingues RC, et al. Pseudoprogression and pseudoresponse: imaging challenges in the assessment of posttreatment glioma. AJNR Am J Neuroradiol 2011;32: 1978-85 CrossRef Medline

10. Hu X, Wong KK, Young GS, et al. Support vector machine multiparametric MRI identification of pseudoprogression from tumor recurrence in patients with resected glioblastoma. J Magn Reson Imaging 2011;33:296-305 CrossRef Medline

11. Wang S, Kim SJ, Poptani H, et al. Diagnostic utility of diffusion tensor imaging in differentiating glioblastomas from brain metastases. AJNR Am J Neuroradiol 2014;35:928-34 CrossRef Medline

12. Wang S, Kim S, Chawla S, et al. Differentiation between glioblastomas, solitary brain metastases, and primary cerebral lymphomas using diffusion tensor and dynamic susceptibility contrast-enhanced MR imaging. AJNR Am J Neuroradiol 2011;32:507-14 CrossRef Medline

13. Wang S, Kim S, Chawla S, et al. Differentiation between glioblastomas and solitary brain metastases using diffusion tensor imaging. Neuroimage 2009;44:653-60 CrossRef Medline

14. Toh CH, Castillo M, Wong AM, et al. Primary cerebral lymphoma and glioblastoma multiforme: differences in diffusion characteristics evaluated with diffusion tensor imaging. AJNR Am J Neuroradiol 2008;29:471-75 CrossRef Medline

15. Agarwal A, Kumar S, Narang J, et al. Morphologic MRI features, diffusion tensor imaging and radiation dosimetric analysis to differentiate pseudo-progression from early tumor progression. J Neurooncol 2013;112:413-20 CrossRef Medline

16. Lacerda S, Law M. Magnetic resonance perfusion and permeability imaging in brain tumors. Neuroimaging Clin N Am 2009;19:527-57 CrossRef Medline

17. Gasparetto EL, Pawlak MA, Patel SH, et al. Posttreatment recurrence of malignant brain neoplasm: accuracy of relative cerebral blood volume fraction in discriminating low from high malignant histologic volume fraction. Radiology 2009;250:887-96 CrossRef Medline

18. Kong DS, Kim ST, Kim EH, et al. Diagnostic dilemma of pseudoprogression in the treatment of newly diagnosed glioblastomas: the role of assessing relative cerebral blood flow volume and oxygen-6methylguanine-DNA methyltransferase promoter methylation status. AJNR Am J Neuroradiol 2011;32:382-87 CrossRef Medline

19. Tsien C, Galbán CJ, Chenevert TL, et al. Parametric response map as an imaging biomarker to distinguish progression from pseudoprogression in high-grade glioma. J Clin Oncol 2010;28:2293-99 CrossRef Medline

20. Kim HS, Kim JH, Kim SH, et al. Posttreatment high-grade glioma: usefulness of peak height position with semiquantitative MR perfusion histogram analysis in an entire contrast-enhanced lesion for predicting volume fraction of recurrence. Radiology 2010;256: 906-15 CrossRef Medline

21. Baek HJ, Kim HS, Kim N, et al. Percent change of perfusion skewness and kurtosis: a potential imaging biomarker for early treatment response in patients with newly diagnosed glioblastomas. $R a$ diology 2012;264:834-43 CrossRef Medline

22. Mangla R, Singh G, Ziegelitz D, et al. Changes in relative cerebral blood volume 1 month after radiation-temozolomide therapy can help predict overall survival in patients with glioblastoma. Radiology 2010;256:575-84 CrossRef Medline

23. Chowdhary S, Chamberlain M. Bevacizumab for the treatment of 
glioblastoma. Expert Rev Neurother 2013;13:937-49 CrossRef Medline

24. Tumor treating fields therapy for recurrent glioblastoma. Manag Care 2012;21:43-44 Medline

25. Finocchiaro G, Pellegatta S. Perspectives for immunotherapy in glioblastoma treatment. Curr Opin Oncol 2014;26:608-14 CrossRef Medline

26. Boxerman JL, Prah DE, Paulson ES, et al. The role of preload and leakage correction in gadolinium-based cerebral blood volume estimation determined by comparison with MION as a criterion standard. AJNR Am J Neuroradiol 2012;33:1081-87 CrossRef Medline

27. Kim DY, Kim HS, Goh MJ, et al. Utility of intravoxel incoherent motion MR imaging for distinguishing recurrent metastatic tumor from treatment effect following gamma knife radiosurgery: initial experience. AJNR Am J Neuroradiol 2014;35:2082-90 CrossRef Medline

28. Zamecnik J. The extracellular space and matrix of gliomas. Acta Neuropathol 2005;110:435-42 CrossRef Medline
29. Barajas RF Jr, Chang JS, Segal MR, et al. Differentiation of recurrent glioblastoma multiforme from radiation necrosis after external beam radiation therapy with dynamic susceptibility-weighted contrast-enhanced perfusion MR imaging. Radiology 2009;253:486-96 CrossRef Medline

30. Pope WB, Lai A, Mehta R, et al. Apparent diffusion coefficient histogram analysis stratifies progression-free survival in newly diagnosed bevacizumab-treated glioblastoma. AJNR Am J Neuroradiol 2011;32:882-89 CrossRef Medline

31. Xu JL, Li YL, Lian JM, et al. Distinction between postoperative recurrent glioma and radiation injury using MR diffusion tensor imaging. Neuroradiology 2010;52:1193-99 CrossRef Medline

32. Jahangiri A, Aghi MK. Pseudoprogression and treatment effect. Neurosurg Clin N Am 2012;23:277-87, viii-ix CrossRef Medline

33. Aquino D, Di Stefano AL, Scotti A, et al. Parametric response maps of perfusion MRI may identify recurrent glioblastomas responsive to bevacizumab and irinotecan. PLoS One 2014;9:e90535 CrossRef Medline 